\title{
Impacto de la investigación clínica en el desarrollo de un país*
}

\section{Impact of clinical research in the development of a country}

\author{
Dora Inés Molina de Salazar, Germán Camilo Giraldo • \\ Manizales (Colombia)
}

\begin{abstract}
Resumen
Con la creciente aparición de centros de investigación por contratación en Colombia y Sur América, se hace necesario resaltar el por qué de esta situación y las ventajas que trae para cada país, tanto económicos como académicos, y el creciente interés de la industria farmacéutica para llegar más a estos países, donde la cantidad de población, la calidad de la investigación y los costos han sido atractivos. En Colombia existen 108 centros de investigación, 53 afiliados a la Asociación Colombiana de Centros de Investigación; Diabetes y Endocrinología, los temas preferidos por los investigadores en Colombia. (Acta Med Colom 2012; 37: 215-219)
\end{abstract}

Palabras clave: investigación, desarrollo económico, industria farmacéutica, estudios clínicos.

\begin{abstract}
With the increasing emergence of research centers though hiring in Colombia and South America, it is necessary to highlight the reason for this situation and the economic as well as academic benefits that it brings to each country, and the growing interest of the pharmaceutical industry to penetrate more in these countries where the number of people, the quality of research and the costs have been attractive. In Colombia there are 108 research centers, 53 of which are members of the Colombian Association of research centers, being Diabetes and Endocrinology the subjects preferred by researchers in Colombia. (Acta Med Colom 2012; 37: 215-219)
\end{abstract}

Keywords: research, economic development, pharmaceutical, clinical studies.

\author{
* Conferencia Lombana Barreneche dictada \\ durante el XXII Congreso Colombiano de \\ Medicina Interna, llevado a cabo del 11-14 \\ de octubre de 2012 en la Ciudad de Carta- \\ gena de Indias. Bogotá, Colombia. \\ Dra. Dora Inés Molina de Salazar: Inter- \\ nista, Docente Titular Facultad de Ciencias \\ para la Salud, Universidad de Caldas. Inves- \\ tigadora Principal IPS Médicos Internistas \\ de Caldas; Dr. Germán Camilo Giraldo \\ González: Subinvestigador IPS Médicos In- \\ ternistas de Caldas. Manizales (Colombia) \\ Correspondencia. Dra. Dora Inés Molina \\ de Salazar, \\ E-mail: doraines56@gmail.com \\ Recibido: 12/XI/2012 Aceptado: 15/XI/2012
}

La ciencia no podría existir, sin escribir. Las características que convierten a la ciencia en ciencia, es que es pública, objetiva, reproducible, predictiva, acumulativa, sistemática y todos éstos dependen de la comunicación escrita. La publicación es el estadio final en la investigación, si ésta no se lleva a cabo en un ámbito científico, la investigación nunca existió; el concepto de medicina basada en la evidencia también depende del escribir,y se convierte en medicina basada en la literatura, pero para que esto se lleve a cabo no se trata tan sólo de escribir, se trata de una correcta investigación con sus protocolos y métodos analíticos (1).

Divididos en cuatro grandes categorías de investigación, los estudios científicos son aquellos que buscan describir, explicar, predecir o manipular el mundo médico, y desde hace cinco siglos se dividen en artículos científicos de tipo observacional, teórico, experimental, metodológico, artículos de revisión y otras formas de comunicación escrita (1). Sin desconocer que todos son fundamentales en el proceso investigativo son los estudios experimentales los que más involucran la participación de la población proporcionando un acceso a nueva información y tecnología, además de generar, empleo e ingresos económicos (2); sin embargo, para los fines de esta revisión es importante tener claro cuáles son los tipos de investigaciones clínicas que se llevan a cabo en el país y en el mundo.

Por un lado está la investigación en salud de los entes educativos o fomentada por el Estado, en nuestro caso la mayor es por Colciencias, en la cual pueden participar entes públicos o privados y por otro la investigación contratada, generalmente patrocinada por las grandes multinacionales, dirigidas por ellos mismos o llevada a cabo por instituciones de investigación por contrato o CRO. Esta última es muy importante ya que hace que ingresen al país inversiones del extranjero y es sobre la cual haremos énfasis a continuación.

Diversos países del mundo y algunos de América Latina están abriendo sus puertas y realizando fértil el terreno para que estos beneficios lleguen a cada país, siendo algunos más aventajados en este tema (2). 
Dando una vista por algunos países a nivel mundial, encontramos el ejemplo de Turquía, donde se habló de investigación mucho antes de la aparición de la declaración de Helsinki o del informe Belmont, su régimen legal hablaba de que para el uso de medicamentos experimentales se debía obtener primero el permiso del paciente (3). En Dinamarca, se conducen cerca de 300 estudios cada año de manera constante, todo esto gracias a que desde 2002 se realizó la vinculación a los lineamientos regulatorios de la Unión Europea. Cuentan en este país, con tres hospitales con unidades completas de buenas prácticas clínicas, que asesoran a otros centros de servicios en investigación. Dinamarca tiene un fuerte sistema para garantizar que la investigación clínica se lleve a cabo en una forma ética e intachable. Todos los pasos necesarios se toman para garantizar la seguridad y los derechos de los sujetos que deciden participar en un estudio clínico (4). En Alemania, cuando se planea una investigación, la primera pregunta es ¿cuánto tiempo tomará el inicio de esta misma?, pues los dos principales elementos que se manejan son las aprobaciones del Comité de Ética (CE) y de la autoridad competente (AC). Sin estas autorizaciones no puede ser iniciado el proceso y es el patrocinador quien se encarga de esto.

Los CE están dirigidos en los hospitales universitarios por los investigadores y los AC son dos, el BfArM y el PEI. El costo de su estudio varía dependiendo de la naturaleza del producto medicinal, y cuando hay modificaciones genéticas en organismos el costo es el más elevado (5). En Suiza, todas las investigaciones clínicas con medicamentos en humanos se deben regir a las buenas prácticas clínicas (BPC) y como premisa esencial tiene el consentimiento informado escrito con todos sus componentes, y en segundo lugar, que exista un seguro para cubrir las posibles complicaciones que se presenten en el estudio (6). En Israel, de los 29 hospitales existentes diez tienen asociación con facultades de medicina y con aproximadamente 20000 médicos con una gran influencia de la medicina norteamericana. El número de estudios clínicos a partir de las diversas instituciones médicas en todo Israel está aumentando cada año. En 2004, había 2099 solicitudes revisadas por las juntas de revisión institucional (IRB) a través de Israel. En cuanto a la investigación médica que involucre la participación de los israelíes, el país incorporó en 1980 los principios de la declaración de Helsinki y la división de medicamentos del Ministerio de Salud publicó las guías, para la conducción de estudios clínicos en humanos. El investigador presenta directamente el estudio clínico al IRB, si es aprobado pasa al ministerio de Salud y si no es detenido y pasa a consideración del director del hospital para la decisión sobre su ejecución. Israel es catalogado como una nación atractiva para la industria farmacéutica, primero porque su población tiene orígenes en varios países y múltiples etnias y segundo porque su sistema de salud esta unificado, resultando muy cómodo hacerle seguimiento a la evolución clínica de los pacientes en cualquier lugar (7). Es Polonia uno de los principales países de la región centro-este europea, para el desarrollo de estudios clínicos, con una población de más de 38 millones de habitantes, la gran mayoría sin tratamientos previos de base, con investigadores altamente calificados y motivados y menores costos en comparación con países de occidente (8). En la India, país de más de mil millones de habitantes, 29 Estados y 22 lenguas oficiales (844 dialectos) el camino a la investigación mejoró en el año 2005, cuando se reemplazó el acta de medicamentos y cosméticos, que sólo permitía estudios en fase II, autorizando también estudios fase III, como en otras partes del mundo. En el pasado existió un sesgo y rechazo de la población por los ensayos clínicos, pues, anteriormente, algunos patrocinadores de los ensayos utilizaron pacientes pobres y analfabetas (población vulnerable), a menudo sin su consentimiento. Afortunadamente, con las nuevas regulaciones, las investigaciones están aumentando y las organizaciones no gubernamentales (más de dos millones en toda India) están actuando como "perros guardianes" o defensores del pueblo (9).

El actual auge de los estudios clínicos llevados a cabo en América del Sur podría dar la impresión de que la investigación clínica se introdujo en el continente recientemente, pero en realidad interesantes investigaciones empezaron a llevarse a cabo en Sur América en las primeras décadas del siglo 20, donde los investigadores eran principalmente científicos europeos, que llegaron como visitantes o inmigrantes y decidieron dedicar sus vidas al estudio de enfermedades endémicas; algunos expertos locales también participaron en estos esfuerzos pioneros, que como resultado produjeron progresos considerables en el conocimiento de la fiebre amarilla, la leishmaniasis y la enfermedad de Chagas, entre otras. Después de la Segunda Guerra Mundial, cuando la investigación médica y de medicamentos nuevos tomaba auge en otros lugares, América del Sur seguía siendo un participante periférico (10).

En Colombia la investigación contratada inició a principio de los años 80 (16). Para esa época casi todas las inversiones de los EE.UU. estaban destinadas a los laboratorios nacionales y de Europa.

Pero ¿qué fue lo que cambió en los últimos 20 años para que se condujeran más estudios clínicos en América del Sur?

1. Estabilización de la política de los países latinoamericanos con un predominio de la democracia.

2. Se logró un desarrollo económico significativo, con menos inflación, una mayor apertura y, por así decirlo, un poco de simpatía más por el capital internacional.

3. Mejoró la parte cientificomédica con el incremento de la cooperación científica internacional contando con centros académicos, con personal capacitado en investigación clínica que llamaron la atención de las filiales locales y de las industrias farmacéuticas.

Como consecuencia de ello, a mediados de 1980, la mayoría de los países desarrollaron normas nacionales para los ensayos clínicos haciendo que el número de estudios creciera de forma constante. Para esa época, la mayoría de 
los países de Sur América no se habían adherido a la conferencia de armonización en estandarización farmacéutica; sin embargo, en 2001 la Organización Panamericana de la Salud estableció un grupo de trabajo (Conferencia Internacional de Armonización de Buenas Prácticas Clínicas 1993), para dar a conocer las buenas practicas clínicas de investigación BPC (GCP good clinical practice) y como resultado de ello se produjo un documento de BPC de las Américas en 2005.

Antes del año 2000, diez de los 12 países tenían regulaciones nacionales incipientes para la práctica de la investigación clínica contratada.

Algunas de las áreas que más impedían la adopción eficiente de las normas de las BPC y que algunas todavía existen eran:

1. La incapacidad de las autoridades sanitarias para garantizar el pleno cumplimiento de las normas.

2. La falta de conocimiento y entrenamiento de las autoridades sanitarias.

3. La falta de conocimiento y entrenamiento académico a nivel de los investigadores y/o comités de ética.

4. Los engorrosos procesos burocráticos para aplicar, iniciar y llevar a cabo estudios clínicos.

5. La falta de entrenamiento en BPC.

Por otra parte, el ciclo de aprobación de un protocolo en algunos de los países de Latinoamérica era relativamente lento, como por ejemplo Brasil.

Por el contrario, Colombia viene trabajando para ser uno de los mas rápidos, el gobierno y el sector privado trabajan juntos y están luchando para poder tener más ensayos en el país (10).

Muchas empresas de consultoría han elaborado informes que muestran América Latina como un destino interesante para la externalización de actividades y ensayos clínicos. Todos ellos destacan los costos favorables, las capacidades lingüísticas, el gran volumen de población, la capacitación del recurso médico y la proximidad cultural con los países occidentales $(10,16)$.

A.T. Kearney ha publicado un índice llamativo para escoger dónde llevar los ensayos clínicos y compara un conjunto de 15 países en el mundo. Brasil ocupa el cuarto lugar en la lista, sobre todo debido a un gran grupo de pacientes y sus bajos costos y Argentina ocupa el séptimo lugar (11).

Hasta septiembre de 2012, los países de América Latina con más ensayos clínicos eran Brasil (3026), México (1711) y Argentina (1424), justo detrás de ellos está Chile (767), Perú (626) y Colombia (576) (17) (Figura 1). En estos países más activos, los reglamentos de los ensayos clínicos se basan en las directrices de la Conferencia Internacional de Armonización (ICH International Conference Harmonization) de las buenas prácticas clínicas (BPC) (12).

Varias cualidades intangibles relacionadas con la investigación clínica contratada son únicas para

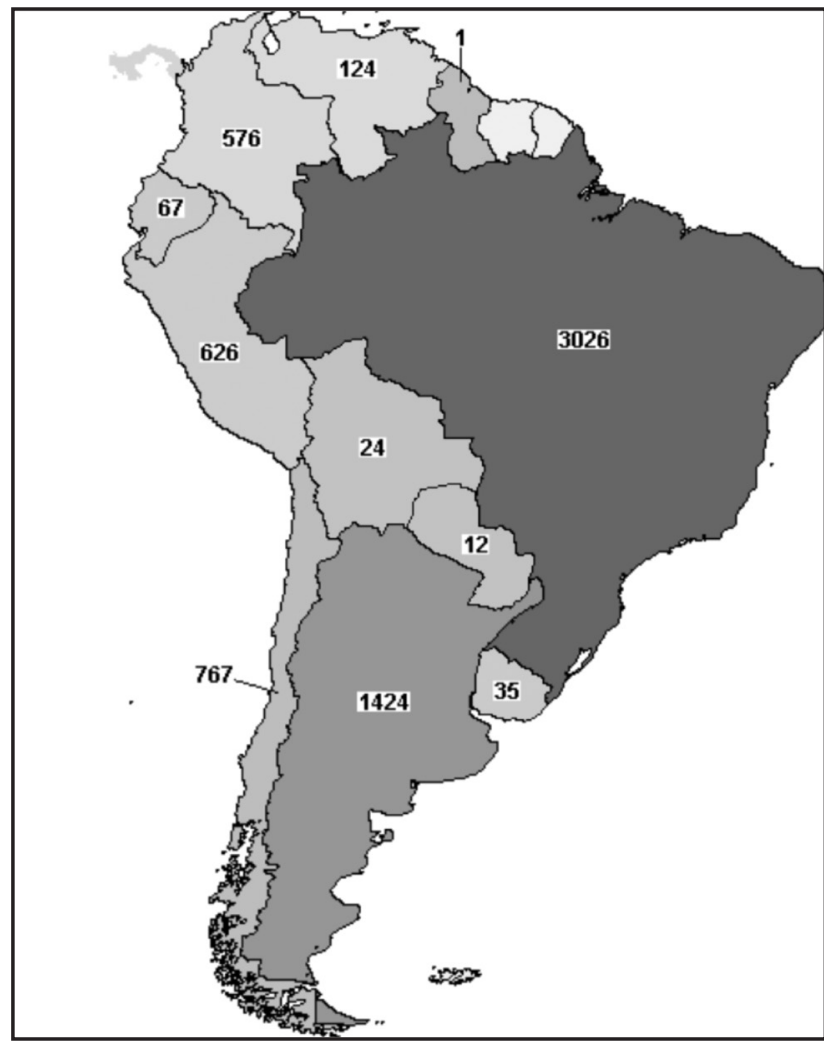

Figura 1. Tomada de mapa de distribución de ensayos clínicos por país. (Sitio de Internet) Disponible en http://clinicaltrials.gov/ct2/search/map. (17)

la región. En primer lugar, las relaciones médicopaciente son muy fuertes, y la mayoría de los pacientes están invitados a participar en los ensayos por sus propios médicos y no reclutados a través de anuncios sin que esto ultimo sea malo. En segundo lugar, la motivación y la apertura del personal de investigación es una característica notable de los muchos sitios en los que realizan un número limitado de estudios, a menudo con los jóvenes y personal en formación. Incluso con los investigadores, la alta motivación es una característica muy importante para superar los problemas relacionados con la infraestructura y la contratación (10).

En Latinoamérica cabe resaltar en el campo de la investigación clínica a Perú, que se ha convertido en una plaza muy atractiva para la inversión de capitales extranjeros y nacionales debido a la mejora de las circunstancias económicas y sociales favorables para la misma. El gobierno peruano cumple una labor activa en la creación de condiciones que promuevan la inversión. Por ello, considerando que la investigación proporciona acceso a nueva información y tecnología, generando, durante su realización, empleos e ingresos económicos; el gobierno publicó un conjunto de disposiciones legales que regulan la ejecución de ensayos clínicos (EC) 
dentro del contexto de las normas internacionales de bioética y buenas prácticas clínicas (13). Sin embargo, al observar de cerca las amplias ventajas económicas y académicas que trae para un país atraer la inversión extranjera, Chile hizo un despliegue amplio de medidas con resultados efectivos en corto plazo. Chile está particularmente interesado en las inversiones destinadas a desarrollar la investigación clínica farmacéutica.

Para ello, el gobierno de Chile está desarrollando un modelo operativo diseñado para cerrar la brecha en comparación con los mejores mercados de ensayos clínicos en el mundo.

En línea con este objetivo, el Ministerio de Salud chileno está poniendo en marcha todos los elementos de un entorno de clase mundial regulatorios, incluyendo directrices internacionalmente reconocidas de comités de ética. En este sentido, CORFO (Agencia para el Desarrollo Económico de Chile) ha desarrollado un conjunto de incentivos financieros para atraer iniciativas de investigación clínica. En 2007, 120 ensayos clínicos fueron aprobados por el Instituto de Salud Pública y según el Instituto de Salud Pública el tiempo para analizar y aprobar un estudio es de 30 días. Aprovecha además todos sus galardones para tratar de atraer la mayor cantidad de estudios como la otorgada por el Foro Económico Mundial que en el año 2006 ubicó a Chile como la economía más competitiva de América Latina (19).

La investigación en salud, en el marco de los sistemas de salud en el mundo, ha sido materia de muchas reuniones internacionales, declaraciones, pronunciamientos e investigaciones sobre la materia. Probablemente uno de los más importantes lo constituye el "reporte 10/90 sobre la investigación en salud". En el cual estiman que los sectores públicos y privados gastan globalmente 56 mil millones de dólares anualmente en este tipo de investigación; sin embargo, menos del $10 \%$ de este monto se destina al $90 \%$ de los problemas de salud del mundo, situación calificada como el desequilibrio 10/90. También concluye que los países con rentas medias y bajas, agrupan $85 \%$ de la población mundial y soportan $92 \%$ de la carga de la enfermedad, mientras que los países ricos agrupan $15 \%$ de la población en el mundo y subsecuentemente soportan $8 \%$ de la carga de la enfermedad, y algunos sectores sostienen que esto se debe a la falta de procesos de acción colectiva que organicen la interacción entre los actores, la dinámica de los procesos y las reglas de juego para la investigación no contratada.

Para el caso colombiano, en el reconocimiento de la brecha 10/90 persisten aún desequilibrios. En el año 2004, con la iniciativa de Colciencias y del Instituto de Salud Pública, de la Universidad Nacional de Colombia, se hizo manifiesto el interés por construir una agenda nacional de prioridades de investigación en salud (no contratada). Participaron representantes de las comunidades científicas en salud. Se organizaron dos reuniones nacionales, dos reuniones regionales, y un foro virtual. Además, se emplearon las tres metodologías mencionadas anteriormente. La complejidad de las metodologías y del proceso de priorización se hizo evidente en las dificultades que tuvieron algunos investigadores en el uso de los instrumentos diseñados para tal fin; debido a la relativa novedad de este tipo de procesos en Colombia, son escasos los antecedentes y experiencias previas al respecto (14).

En cuanto a la investigación no contratada en Colombia, para el año 2010, Colciencias reportó un incremento en $20.07 \%$ en el número de grupos registrados en la Plataforma ScienTI - Colombia. Se incrementó en $23.72 \%$, los grupos de investigación que cumplen la definición. Se incrementó en $15.06 \%$ los grupos de investigación clasificados y un aumento en la calidad de la producción científica del país (18).

En Colombia se realizan estudios clínicos principalmente en fases II, III y IV, patrocinados por las compañías de investigación y desarrollo y a través de la contratación de CROs,(Organización de Investigación por contrato u Organización de investigación clínica) y centros de investigación especializados para la investigación no contratada.

La medición del número de ensayos clínicos en un país y su evolución cualitativa nos puede dar una idea del grado de desarrollo de la investigación clínica en el mismo, de la calidad de la asistencia sanitaria y de la consideración que tiene dicho país por la industria farmacéutica.

Pero el número de estudios clínicos en gran medida depende de la confianza y la seguridad tanto jurídica como técnica que se tiene en el país por parte de las casas matrices y las CROs. En Colombia hay un gran avance en los últimos años y el Invima ha expedido en 2008 la norma por la cual se adoptan las buenas prácticas clínicas para las instituciones que conducen investigación con medicamentos en seres humanos (Resolución 2378) y desde entonces se ha venido realizando un trabajo conjunto con AFIDRO, mediante conversatorios y mesas de trabajo para garantizar su adecuada implementación con el apoyo de la ACIC (Asociación de Centros de Investigación Clínica) y AVANZAR (Asociación para el Avance de la Investigación en Colombia). Esto ha permitido que los ojos estén puestos en el país y que sea de gran interés por parte de las compañías y de CROs de trayectoria internacional que han empezado a instalarse en Colombia, favoreciendo el crecimiento del sector de la investigación clínica.

En el país ha mejorado el tiempo en que se aprueba un protocolo aunque aún existen dificultades importantes, lo que puede llegar a desincentivar la inversión por parte de casas matrices, tal es el caso de reporte de eventos adversos que en los últimos seis meses se ha venido discutiendo dadas las exigencias imposibles de cumplir que se pretenden im- 
poner a quienes hacen investigación y aún no se ha aclarado la norma para su aplicación, siendo el principal problema los tiempos de aprobación del Invima.

Cabe resaltar la importancia de la investigación en el desarrollo de medicamentos innovadores y su repercusión en la gestión sanitaria y clínica: "El conocimiento debe ser el objetivo fundamental de la investigación, contemplada como elemento de estímulo para los profesionales, y de prestigio para los centros". La atención sanitaria y la investigación biomédica constituyen demandas sociales prioritarias." En 2009 a escala mundial: 65,2 billones de dólares se invirtieron en investigación contratada, los centros de investigación -IPS se benefician con la incorporación de conocimientos disponibles en países más avanzados y adquisición de tecnologías, procesos y equipos de última generación (15).

En Colombia a la fecha y según la página publicada por el Invima existen 108 centros de investigación (85 en 2011 y 23 en 2012), que han cumplido con la certificación en buenas prácticas clínicas conforme a la ley, de esos, 53 se encuentran afiliados dentro de la Asociación Colombiana de Centros de Investigación Clínica (ACIC). Existen aún 16 centros en espera de la visita del Invima entre el Eje Cafetero, Cali y Bogotá. En cuanto al tema de la generación de oportunidades laborales se estiman cerca de 5000 empleos en los centros de investigación afiliados a la ACIC, y de estos mismos centros se encontró que dentro de las áreas de preferencia para la investigación clínica contratada están la diabetes y endocrinología reportada por 20 centros, la cardiología por 19, la oncología por 13 centros, igual número que se dedican además en áreas de infectología y vacunación, nueve centros entre enfermedad pulmonar obstructiva crónica y neumología e igual cantidad en reumatología, ocho en medicina interna y seis centros reportaron respectivamente todas las especialidades médicas, hematología y oftalmología. De ahí en menores proporciones podemos encontrar estudios en las áreas de la ginecología, pediatría, psiquiatría, dermatología, neurología, nefrología y gastroenterología (16).

\section{Para el futuro}

- Se requiere generar las condiciones para que en el país se pueda desarrollar todo el proceso que va desde la investigación básica, los estudios preclínicos y los estudios clínicos hasta la producción.

- Los centros de investigación y las universidades deben generar conocimiento a partir de la inversión pública/ privada.

- Se debe obtener una estandarización de los centros de investigación y propugnar por centros de excelencia

- Elaborar propuestas que surjan de necesidades reales del sector social y económico en asocio con el sector académico, para lograr una racionalización en términos de inversión.

- Consolidar un ambiente de seriedad y seguridad jurídica en el marco de BPC, (15)
- El apoyo de todo el grupo médico nacional, ya sea con el aporte de pacientes para los estudios o con la generación de centros de investigación con todo el marco de la ley, para llamar aún más la atención de más casas matrices.

- Animar por medio de un terreno para la investigación seguro sin trabas, a los ejecutivos colombianos que laboran en grandes multinacionales para que puedan vender con más ahínco nuestro país como un escenario confortable para la investigación.

- Sería interesante, la unificación del sistema en el cual se manejan las historias clínicas de los pacientes, no sólo con fines de investigación, sino también ahorraría grandes costos en el sistema de salud en cuanto a antecedentes y apoyos diagnósticos realizados en general, evitando incluso en muchas circunstancias repetir estos mismos.

\section{Fuentes de financiación}

La Dra. Dora Inés Molina declara no tener conflictos de interés de ningún tipo así como niega haber recibido algún tipo de honorario o patrocinio por la elaboración de este artículo.

El Dr. Germán Camilo Giraldo declara no tener conflictos de interés de ningún tipo así como niega haber recibido algún tipo de honorario o patrocinio por la elaboración de este artículo.

\section{Referencias}

1. Lang TA. How to write, publish, and present in the health sciences: a guide for clinicians and laboratory researches. United States of America: ACP 2010.

2. Instituto Nacional de Salud (INS). Perú: un país de oportunidades en investigación clínica. Oficina General de Investigación y Transferencia de Tecnología (OGITT). Lima: 2010

3. Akan H, Ílbars H, Ayhan I, MD, Kansu E, Akova M, et al. New GCP rules in Turkey. Monitor 2007; 2: 41-44.

4. Beck GR. An Overview of Clinical Studies in Denmark. Monitor 2007; 2: 40.

5. Buck K. Starting clinical trials in Germany. Monitor 2007; 2: 31-33.

6. Felenda J. Submission of a clinical trial in switzerland. Monitor 2007; 2: 47-50.

7. Koren A. Successful Start of Clinical Trials in Israel A Roadmap for Pharmaceutical and Medical Device Trials. Monitor 2007; 2: 57-61.

8. Kozak M. Study startup in Polonia. Monitor 2007; 2: 23-26.

9. Kumar A. The clinical trials road in India looping for the land minds. Monitor 2007; 2: 17-21.

10. Motti E. Doing Clinical Research in South America The Implications of Good Clinical Practice. Monitor 2008; 63-67.

11. Kearney AT. Destination Latin America: A Near-Shore Alternative. Available at http://www.atkearney.com/shared_res/pdf/ Near-Shore_Latin_America_S. pdf; accessed Feb. 2008.

12. Blanchard A. Clinical research in Latin America, an updated preview of rules and regulations. Monitor 2012; 26(5): 27-32.

13. Yagui M, Espinoza M, Caballero P, Castilla T, Garro G, Yamaguchi L, et al Avances y retos en la construcción del sistema nacional de investigación en salud en el Perú. Rev Peru Med Exp Salud Publica 2010; 27(3): 387-97.

14. Escobar FA, Agudelo CA. Evaluación del Proceso de Priorización en Salud en Colombia. Perspectiva de Grupos de Investigación Ubicados en Bogota. Rev salud publica 2009; 11(2): 212-224,

15. Asociación de laboratorios farmacéuticos de investigación y desarrollo, Afidro. Talking Points Estudios Clínicos en Colombia.

16. Asociación colombiana de centros de investigación, ACIC.

17. Mapa de distribución de ensayos clínicos por país. (Sitio de Internet). Disponible en: http://clinicaltrials.gov/ct2/search/map. Revisada en Septiembre de 2012.

18. Castro L. Informe ejecutivo de la Convocatoria Nacional para la Medición de Grupos de Investigación, Tecnológica o de Innovación Año 2010. (sitio en Internet). Disponible en:http://www.colciencias.gov.co/sites/default/files/ckeditor_files/files/Informe\%20corto\%20de\%20los\%20resultados-vf.pdf Revisada en Septiembre de 2012

19. Ministerio de salud. Chile: un lugar emergente para los ensayos clínicos. Agencia Chilena para el desarrollo económico, CORFO. Chile: 2008. 